\title{
FUNIL DE HAINES MODIFICADO: CURVAS DE RETENÇÃO DE SOLOS PRÓXIMOS À SATURAÇÃO(1)
}

\author{
Fabio Augusto Meira Cássaro ${ }^{(2)}$, Luiz Fernando Pires ${ }^{(2)}$, Rosiane \\ Antures dos Santos ${ }^{(3)}$, Daniel Giménez ${ }^{(4)} \&$ Klaus Reichardt ${ }^{(5)}$
}

\section{RESUMO}

O movimento da água através da matriz do solo é geralmente modelado pelas equações de Darcy, Darcy-Buckingam e Richards, cujo uso está baseado no conhecimento de algumas propriedades físicas do solo, como, por exemplo, a distribuição de poros e a retenção de água pelo solo. A retenção de água pelo solo é conhecida a partir da determinação de sua curva de retenção $(\mathrm{CR})$ ou curva característica. O primeiro objetivo deste trabalho foi apresentar um aparato simples, desenvolvido pela modificação do funil de placa porosa (funil de Haines), para a investigação e o levantamento de CRs detalhadas, em amostras de solos em condições de umidade próximas à saturação e em amostras com potenciais mátricos $\left(\Psi_{\mathrm{m}}\right)$ que vão desde $0 \mathrm{kPa}$ a aproximadamente $-12 \mathrm{kPa}(120 \mathrm{~cm}$ de altura de coluna de água). Foram investigados agregados de um Latossolo Vermelho distrófico (LVd) de uma região do Estado do Paraná, cujos diâmetros médios e densidades variaram, respectivamente, entre 1,6 e $5,7 \mathrm{~cm}$ e 1,01 e $1,31 \mathrm{~g} \mathrm{~cm}^{-3}$, e amostras arenosas reconstituídas com areias de diâmetros médios entre $0,106 \mathrm{~mm}$ e $2,000 \mathrm{~mm}$, com dimensões fractais de fracionamento $\left(D_{f}\right)$ entre 2,5 e 3,0. O segundo objetivo do trabalho foi inferir a distribuição de poros das amostras investigadas. Isso foi conseguido utilizando-se os parâmetros de ajuste da curva de van Genuchten aos pontos das CRs obtidas para a determinação da Função Capacidade de Água (FCA). Pela análise dessas FCAs, observou-se que as amostras de agregados de solo apresentaram um sistema poroso de maior complexidade; e que a variação da granulometria do solo arenoso está diretamente relacionada às modificações de

\footnotetext{
(1) Recebido para publicação em julho de 2007 e aprovado em outubro de 2008.

(2) Professor Adjunto, Departamento de Física (DEFIS), Universidade Estadual de Ponta Grossa - UEPG. Av. Carlos Cavalcanti 4748, Uvaranas, CEP 84031-900 Ponta Grossa (PR). E-mail: fcassaro@uepg.br

(3) Mestre em Física, Programa de Pós-Graduação em Ciências (Área de Concentração: Física), DEFIS/UEPG. Bolsista CAPES.

(4) Associate Professor, Department of Environmental Sciences, Rutgers University, 14 College Farm Road, New Brunswick, NJ 08901-8551, USA. E-mail: gimenez@envsci.rutgers.edu

(5) Professor Titular da Universidade de São Paulo - USP. Av. Centenário 303, Agronomia, CEP 13400-970 Piracicaba (SP). Email: klaus@cena.usp.br
} 
suas propriedades de retenção de água. Finalmente, a variação na densidade dos agregados investigados não alterou significativamente o comportamento das curvas de retenção na faixa de tensões estudadas. Termos de indexação: van Genuchten, curva de retenção, Função capacidade de
água.

\title{
SUMMARY: MODIFIED HAINES' FUNNEL: SOIL WATER RETENTION CURVES OF SOIL SAMPLES NEAR SATURATION
}

\begin{abstract}
Water movement through the soil matrix is generally modeled using equations of Darcy, Darcy-Buckingham and Richards, which are based on the knowledge of some soil physical properties such as, soil pore size distribution and water retention characteristics. Soil water retention is determined by the soil water retention curve (SWRC) of the soil. The first objective of this study is to develop a simple apparatus by a modification of the Haines' funnel, to obtain detailed SWRC of near-saturation soil samples, samples in matric potentials $\left(\psi_{\mathrm{m}}\right)$ varying from 0 to $-12 \mathrm{kPa}$ (water column $120 \mathrm{~cm}$ ). Soil aggregates of a Dystrophic Red Latosol (LVd) from Paraná were investigated, with an average diameter between 1.6 and $5.7 \mathrm{~cm}$, soil bulk density between 1.01 and $1.31 \mathrm{~g} \mathrm{~cm}^{-3}$, respectively, and reconstituted sand samples, with a mean sand size of $0.106 \mu \mathrm{m}$ to $2.000 \mathrm{~mm}$, with fractal fragmentation dimensions $\left(D_{f}\right)$ varying from 2.5 to 3.0. A second objective was to analyze the soil pore size distribution of the soil samples. For this purpose the van Genuchten interpolation equation parameters were used to establish the water storage function (WSF) of the samples. In conclusion, an analysis of the WSFs showed that the soil aggregates had a more complex soil pore system; that the soil particle distribution of the sandy soil is directly related to their water retention properties; and finally, that bulk density did not significantly affect soil water retention of the soil aggregates.
\end{abstract}

Index terms: van Genuchten, aporosity, WSF.

\section{INTRODUÇÃO}

De acordo com a SSSA (Soil Science Society of America), um agregado de solo é definido como um grupo de partículas primárias unidas mais fortemente que as partículas que o circundam (SSSA, 1997). Agregados de solos têm sido estudados em termos de sua distribuição de tamanhos na matriz do solo, estabilidade, densidade e resistência (Dexter et al., 1984; Amézketa, 1999). Entretanto, há pouca pesquisa sobre as propriedades hidráulicas dos solos em nível de agregados (McKenzie \& Dexter, 1996; LeedsHarrison \& Youngs, 1997) e também pouca investigação no sentido de relacionar informações a respeito da estrutura do solo ou do agregado com suas propriedades hidráulicas.

O estudo do movimento da água no solo se faz por meio de operações que envolvem o conteúdo e a energia da água nesse meio poroso. As equações de DarcyBuckingham e de Richards são exemplos de equações que envolvem esses conceitos; suas soluções dependem, entre outras coisas, do conhecimento da curva de retenção da água pelo solo (CR). Quando o conteúdo de água no solo se encontra próximo à saturação (potenciais mátricos mais altos), a CR depende basicamente do arranjo poroso do solo, ou seja, de sua estrutura. À medida que o solo perde umidade, a densidade e a porosidade deste passam a ter pouca importância no comportamento da CR (Reichardt \& Timm, 2004).

A determinação adequada de uma CR é de suma importância para implementação e manejo de práticas de cultivo agrícola. No entanto, sua determinação consome muito tempo e está sujeita aos processos de histerese (Klute, 1986), fazendo com que muitos pesquisadores propusessem modelos de previsão da CR com base em propriedades físicas do solo. Haverkamp \& Parlange (1986) apresentam um modelo simples para a predição das características da CR de solos arenosos, com base em propriedades texturais e estruturais do solo, as quais podem ser determinadas em laboratório, sem muita dificuldade.

Nas últimas décadas, diversos modelos matemáticos foram desenvolvidos para se estimar o movimento da água no solo a partir da CR (Brooks \& Corey, 1964; van Genuchten \& Leij, 1992; Poulsen et al., 1998). Esses modelos foram criados pelo fato de que medidas diretas do deslocamento da água no solo 
são geralmente trabalhosas, caras e consomem bastante tempo. Alexander \& Skaggs (1986) apresentaram um modelo teórico para a estimativa da condutividade hidráulica do solo não-saturado a partir de dados da CR e da consideração de que o solo é um conjunto de capilares, pelos quais a água flui.

Zhuang et al. (2001) desenvolveram um modelo teórico para estimar a condutividade hidráulica do solo não-saturado a partir de algumas propriedades físicas do solo e compararam seus resultados com os obtidos por outros cinco modelos. Um dos principais resultados obtidos por esses autores foi a importância de incorporar dados de densidade e textura do solo aos modelos de obtenção da condutividade hidráulica do solo a partir de CRs.

Na Ciência do Solo, é cada vez mais comum a utilização de modelos baseados na geometria fractal para a modelagem das propriedades hidráulicas do solo (Mandelbrot, 1967). Esses modelos são obtidos assumindo propriedades de auto-similaridade para: tamanhos de poros, rugosidade dos poros e complexidade dos caminhos porosos (Tyler \& Wheatcraft, 1989; Fuentes et al., 1996). Usualmente, esses modelos possuem uma lei de potência associada a eles, sendo o expoente desta lei a dimensão fractal (D), a qual se relaciona a atributos geométricos da estrutura do solo. Essa dimensão fractal é um índice estatístico que tem sido utilizado com bastante sucesso na caracterização dos espaços porosos inter e intraagregados (Hatano et al., 1992; Anderson et al., 1996).

Dimensões fractais, obtidas a partir da análise da estrutura do solo, vêm sendo comparadas às Ds utilizadas em modelos para descrever CRs (Comegna et al., 1998). Por exemplo, Crawford et al. (1995) obtiveram estreita correlação entre $\mathrm{D}$ associada à parte porosa do solo e aquela associada às CRs. Perrier et al. (1999), a partir de modelos geométricos baseados na dimensão fractal da fase sólida do espaço poroso do solo, inferiram a respeito de suas propriedades de condução e de retenção de água.

Recentemente, Fuentes et al. (2005) apresentaram modelos fractais para a modelagem da condutividade hidráulica de solos não-saturados a partir do conhecimento de suas CRs. Huang \& Zhang (2005) usaram modelos também baseados na geometria fractal para a modelagem de CRs de solos de textura fina. Pires et al. (2005) desenvolveram um novo método com base em métodos nucleares para a determinação de CRs de um Latossolo VermelhoAmarelo (LVAd) e um Nitossolo Vermelho (NVe) da região de Piracicaba. Este último método possui como principal vantagem a não necessidade de se submeter uma amostra de solo a diversos ciclos de umedecimento e secamento para o levantamento de uma CR.

Resultados conclusivos a respeito da aplicabilidade dos vários modelos que envolvem a estrutura do solo na predição de suas propriedades de retenção dependem de método adequado para o levantamento de CRs dos diversos tipos de solos existentes.

\section{TEORIA}

O funil de Haines, ou funil de placa porosa (Figura 1), é um equipamento utilizado para encontrar a umidade que permanece em uma amostra de solo após a aplicação de uma determinada sucção nela.

Quando um desnível $h$ é gerado entre o tubo flexível e o suporte contendo a placa porosa, a diferença de pressão ocasionada faz com que apareçam meniscos com um determinado valor de raio de curvatura nos poros que constituem a placa porosa. Esse desnível ocorre até um determinado valor máximo ( $\left.\mathrm{h}_{\max }\right)$, em torno de 8,5 m, a partir do qual o menisco é rompido, ocasionando passagem de ar através da placa porosa (Libardi, 2000; Reichardt \& Timm, 2004).

O procedimento para a medida de $\psi_{m}$ por meio de um funil de Haines envolve a colocação de uma amostra saturada em contato hidráulico com a placa porosa, mantendo-se o nível do tubo flexível a uma altura um pouco superior à da amostra. Depois de realizado o contato hidráulico, abaixa-se a mangueira flexível até um valor de $h$ desejado (Figura 1B). Com o desnível, irá ocorrer a drenagem da água presente na amostra de solo (gotejamento através do tubo flexível) até que a condição de equilíbrio seja alcançada, momento em que o gotejamento é cessado. Na condição de equilíbrio, o potencial total $\left(\psi_{\mathrm{t}}\right)$ (a soma dos potenciais de pressão, mátrico e gravitacional, ou seja, $\psi=\mathrm{P}+\psi_{\mathrm{m}}^{6}+\mathrm{Pgz}$ ) em qualquer ponto do lado da solução do solo (ponto A) será igual ao potencial total em qualquer ponto da amostra de solo que agora não se encontra mais saturada. Portanto, observa-se (Figura 1b) que, na condição de equilíbrio, $\psi_{\mathrm{t}}$ em A é igual a $\psi_{\mathrm{t}}$ em B (situado no centro geométrico da amostra):

$$
\psi_{\mathrm{t}}(\mathrm{A})=\psi_{\mathrm{t}}(\mathrm{B})
$$

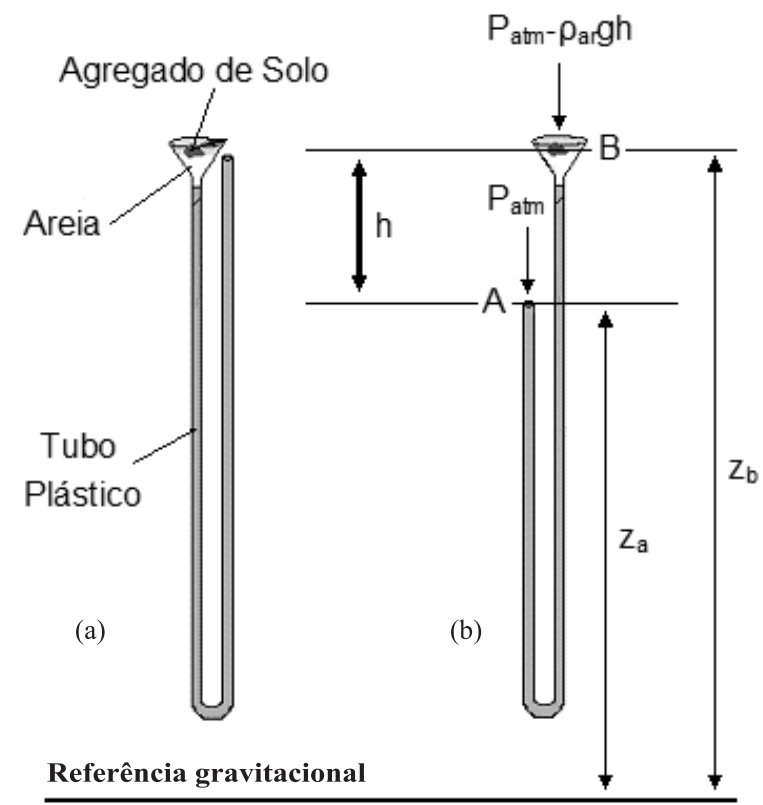

Figura 1. Esquema do funil de Haines modificado: (a) saturação e (b) tensão $h$. 
O potencial total do ponto $\mathrm{A}$ e o do B são, respectivamente, dados pelas seguintes expressões:

$$
\psi_{\mathrm{t}}(\mathrm{A})=\mathrm{P}_{\mathrm{atm}}+\rho_{\mathrm{sol}} \mathrm{gz}_{\mathrm{a}}
$$

e

$$
\psi_{\mathrm{t}}(\mathrm{B})=\left(\mathrm{P}_{\mathrm{atm}}-\rho_{\mathrm{argh}}\right)+\psi_{\mathrm{m}}^{\mathrm{r}}+\rho_{\mathrm{sol}} \mathrm{gz}_{\mathrm{b}}
$$

nas quais os termos referentes aos potenciais de pressão, mátrico e gravitacional são apresentados e $g$ é aceleração da gravidade $\left(\mathrm{m} \mathrm{s}^{-2}\right) ; z$, a posição $(\mathrm{m})$ dos pontos A e B em relação a um referencial arbitrário; $\mathrm{e}$ $\rho_{\text {sol }}$ e $\rho_{\text {ar }}$, as densidades da solução do solo e do ar $\left(\mathrm{kg} \mathrm{m}^{-3}\right)$.

Da igualdade entre as equações (2) e (3) obtém-se:

$$
\frac{\psi_{\mathrm{m}}^{\prime}}{\rho_{\mathrm{sol}}}=-\mathrm{g}\left(\mathrm{z}_{\mathrm{b}}-\mathrm{z}_{\mathrm{a}}\right)+\frac{\rho_{\mathrm{ar}}}{\rho_{\mathrm{sol}}} \mathrm{gh}
$$

de acordo com o que é apresentado na figura $1 b$, $\mathrm{z}_{\mathrm{a}}-\mathrm{z}_{\mathrm{b}}=\mathrm{h}$; dessa forma:

$$
\frac{\psi_{\mathrm{m}}^{\prime}}{\rho_{\text {sol }}}=-\left(\frac{\rho_{\text {sol }}-\rho_{\text {ar }}}{\rho_{\text {sol }}}\right) \mathrm{gh} \cong-\mathrm{gh}
$$

uma vez que $\rho_{\mathrm{ar}}<<\rho_{\text {sol }}$.

A equação (5) representa o potencial mátrico obtido em unidades de energia por volume de solução (Reichardt \& Timm, 2004). Caso essa equação seja dividida pela aceleração da gravidade, o resultado será expresso em unidades de carga hidráulica $(\mathrm{m})$ :

$$
\psi_{\mathrm{m}}=-\mathrm{h}
$$

Assim, uma curva de retenção da água no solo é obtida variando-se o valor de $h$ e medindo-se a umidade que permanece na amostra para cada valor deste $h$ (Libardi, 2000).

\section{MATERIAL E MÉTODOS}

Para realização deste trabalho, foi desenvolvido um sistema idealizado a partir da idéia do funil de Haines, descrito anteriormente, para a determinação de CRs de amostras de diferentes estruturas e estados de agregação, próximas da saturação (Klute, 1986; Libardi, 2000; Reichardt \& Timm, 2004). O sistema consiste de um funil preenchido com areia, ao qual se conectou uma mangueira flexível em sua extremidade inferior (Figura 1a).

O que diferencia este sistema do original é a substituição da placa porosa, presente no funil de Haines, por areia com partículas de diâmetro menor que $106 \mathrm{~mm}$, a qual é capaz de suportar potenciais mátricos de aproximadamente $-12 \mathrm{kPa}(-120 \mathrm{~cm}$ de altura de coluna de água). Neste artigo, em algumas passagens $-\psi_{\mathrm{m}}$ será substituído por $h$, pelo fato de $h$ ser positivo para solos não-saturados.
Pelo abaixamento da mangueira em relação à superfície da areia, é possível produzir uma tensão (suç̧ão) $h(\mathrm{~Pa})$ na amostra de solo (Figura 1b). Essa sucção remove água da amostra até que uma condição de equilíbrio, para esta sucção $h$ aplicada, seja estabelecida. Nessa condição, poros que apresentem raio maior que um determinado $r$ serão esvaziados (equação 7):

$$
\mathrm{r}=\frac{2 \sigma \cos \alpha}{\rho \mathrm{gh}}
$$

na qual $\sigma$ é a tensão superficial da água $\left(\mathrm{N} \mathrm{m}^{-1}\right)$ e $\rho$ é a densidade do líquido $\left(\mathrm{kg} \mathrm{m}^{-3}\right)$.

Para evitar a perda de areia do sistema, um papelfiltro foi colocado na extremidade inferior do funil. De acordo com a equação anterior, $\mathrm{h}=12 \mathrm{kPa}$ equivale ao esvaziamento de poros de até $12,2 \mathrm{~mm}$ de raio, relacionando-se com a retenção de água devida aos macroporos do solo. A umidade volumétrica da amostra, para cada valor aplicado de tensão, foi obtida segundo o procedimento usual descrito em Embrapa (1997).

O trabalho foi realizado considerando amostras de solos arenosos, reconstruídas de acordo com distribuições fractais de partículas (Arya \& Paris, 1981; Tyler \& Wheatcraft, 1992; Perfect et al., 1992). Foram investigadas distribuições com dimensões fractais de fracionamento $D_{f}$ (Perfect et al., 1992) variando entre 2,50 e 3,00.

A preparação de uma amostra foi obtida considerando-se a equação (8):

$$
M\left(r_{i}\right)=\frac{r^{-D_{f}}}{\sum_{i} r_{i}^{-D f}} M_{t}
$$

na qual $M_{t}$ e $M\left(r_{j}\right)$ são, respectivamente, a massa total da amostra e a massa das partículas de um determinado tamanho característico $r_{i}$, o qual é determinado pela malha da peneira utilizada para separação das partículas de areia.

Essa equação relaciona $D_{f}$ e a percentagem em massa, $M\left(r_{j}\right) / M_{t}$, da fração de um determinado tamanho característico $\left(r_{i}\right)$ que irá compor uma amostra. Os tamanhos médios das partículas (obtidas por peneiramento) escolhidas para compor as amostras de areia foram de 0,$106 ; 0,256 ; 0,400 ; 1,380$ e $1,500 \mathrm{~mm}$. No quadro 1 é apresentada a percentagem de ocorrência de cada classe de partículas nas amostras de solo investigadas.

Após a mistura das partículas desses diferentes tamanhos, a amostra resultante da mistura foi acondicionada homogeneamente em recipientes cilíndricos de PVC, cujas dimensões aproximadas eram de $5 \mathrm{~cm}$ de altura e $3 \mathrm{~cm}$ de raio.

As CRs das amostras foram obtidas variando-se a sucção aplicada, em intervalos de $1 \mathrm{kPa}$, desde 0 até cerca de $12 \mathrm{kPa}$. A fim de se evitar a evaporação de 
Quadro 1. Distribuição do tamanho de partículas utilizadas na reconstrução das amostras de solo arenoso

\begin{tabular}{|c|c|c|c|c|c|}
\hline \multirow{2}{*}{$\mathbf{d}^{(1)}$} & \multicolumn{5}{|c|}{ Percentagem de ocorrência } \\
\hline & $0,106-0,212$ & $0,212-0,300$ & $0,300-0,500$ & $1,000-1,760$ & $1,000-2,000$ \\
\hline $\mathrm{mm}$ & 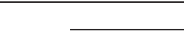 & 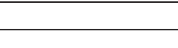 & $\%$ & 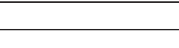 & - \\
\hline $\mathrm{D}_{\mathrm{f}}=2,50$ & 8,43 & 13,10 & 16,37 & 30,41 & 31,70 \\
\hline $\mathrm{D}_{\mathrm{f}}=2,75$ & 13,86 & 16,67 & 18,64 & 25,40 & 25,93 \\
\hline $\mathrm{D}_{\mathrm{f}}=3,00$ & 20,00 & 20,00 & 20,00 & 20,00 & 20,00 \\
\hline
\end{tabular}

(1) $d$ representa a faixa de diâmetros das partículas utilizadas na reconstrução das amostras de solo arenoso com os diferentes valores de dimensão fractal de fracionamento $\left(\mathrm{D}_{\mathrm{f}}\right)$.

água pela superfície das amostras, os funis foram cobertos com plástico durante a determinação de toda uma curva de retenção.

Cada ponto $\left(h_{i}, \theta_{j}\right)$ da CR foi obtido esperando-se um intervalo de tempo de aproximadamente $24 \mathrm{~h}$ entre o início da aplicação da sucção e a retirada da amostra do funil. Esse intervalo de tempo foi considerado suficiente para que se estabelecesse o equilíbrio da água das amostras com a sucção aplicada.

A umidade de saturação $\left(\theta_{\mathrm{s}}\right)$ foi obtida a partir das amostras saturadas $(\mathrm{h}=0 \mathrm{kPa})$ e seguindo método descrito em Embrapa (1997). A umidade volumétrica $\theta_{i}$ foi obtida para cada valor de sucção $h_{i}$ aplicada nas amostras (Embrapa, 1997; Libardi, 2000; Reichardt \& Timm, 2004).

$\mathrm{O}$ ajuste das CRs, para os dados normalizados de umidade $\left(\theta_{\mathrm{i}} / \theta_{\mathrm{s}}\right)$, foi obtido utilizando-se o programa RETC (van Genuchten et al., 1991). Nesse programa, o método dos mínimos quadrados é usado para encontrar os parâmetros da curva (van Genuchten, 1980), equação (9), que melhor se ajusta aos dados experimentais obtidos. Em se tratando dos ajustes de todas as amostras, foi adotada a condição de Mualem, $\mathrm{m}=1-1 / \mathrm{n}$ (Mualem, 1986):

$$
\theta=\theta_{r}+\frac{\left(\theta_{s}-\theta_{r}\right)}{\left[1+\left(\alpha \psi_{m}\right)^{n}\right]^{m}}
$$

em que $\theta_{r}$ representa a umidade residual da amostra de solo, a qual é a umidade remanescente na amostra para a última tensão aplicada $(\mathrm{h}=12 \mathrm{kPa}$ neste estudo); e $\alpha, m$ e $n$ são parâmetros de ajuste da equação.

A partir da obtenção dos parâmetros da equação de van Genuchten (equação 9), a Função Capacidade de Água (FCA) foi obtida da derivada da curva de retenção com relação ao potencial matricial, sendo representada pela equação 10 :

$$
F C A=\frac{d \theta}{d \psi_{m}}=\frac{\alpha\left(\theta_{s}-\theta_{r}\right)(n-1)\left(\psi_{m}\right)^{n-1}}{\left[1+\alpha\left(\psi_{m}\right)^{n}\right]^{2-\frac{1}{n}}}
$$

De acordo com Jury et al. (1991) e Libardi (2000), a FCA fornece uma idéia da distribuição do tamanho dos poros em uma amostra de solo investigada.
Para efeitos de comparação entre as amostras de solo, foi determinada a $F C A$ normalizada $\left(\mathrm{FCA}_{\mathrm{n}}\right)$, sendo obtida por meio da divisão de todos os valores da FCA pela sua área (inserir txt-1):

$$
F C A_{n}=\frac{F C A}{\int_{\text {raios }} F C A d r}
$$

Dessa forma, a condição de normalização implica uma área unitária sob a curva $F C A_{\mathrm{n}}$, pois:

$$
\int_{\text {raios }}\left(F C A_{n}\right) d r=\int_{\text {raios }} \frac{(F C A) d r}{\int_{\text {raios }}(F C A) d r}=\frac{1}{\int_{\text {raios }}(F C A) d r} \int_{\text {raios }}(F C A) d r=1
$$

Com relação aos agregados de solo, estes foram obtidos em posições de coleta escolhidas de forma aleatória, na camada superficial $(0-10 \mathrm{~cm})$ de um campo experimental localizado na região de Ponta Grossa, PR, cujo solo é caracterizado como um Latossolo Vermelho distrófico (LVd). Os métodos usados para sua obtenção em campo, determinação de seus volumes e de suas densidades globais encontram-se disponíveis nos endereços eletrônicos (Soil Aggregates, 2005) listados na literatura citada deste trabalho.

\section{RESULTADOS}

As CRs das amostras de solo arenoso, reconstruídas a partir da variação da $\mathrm{D}_{\mathrm{f}}$, encontram-se na figura 2 .

No quadro 2 são apresentadas algumas características físicas das amostras de solo arenoso utilizadas nos experimentos, bem como os valores dos parâmetros obtidos pelo ajuste com a equação de van Genuchten.

Os parâmetros das curvas de retenção obtidos para as amostras de diferentes valores de $D_{f}$ foram usados na determinação das $F C A_{n} \mathrm{~s}$ (Figura 3).

Os parâmetros de ajuste obtidos das CRs dos agregados de solo foram posteriormente utilizados na determinação de suas $F C A_{n} \mathrm{~s}$. As $C R$ s e as $F C A_{n}$ s para os agregados estão apresentadas, respectivamente, nas figuras 4 e 5. As características físicas e os parâmetros de ajuste do modelo de van Genuchten para os agregados encontram-se no quadro 3. 


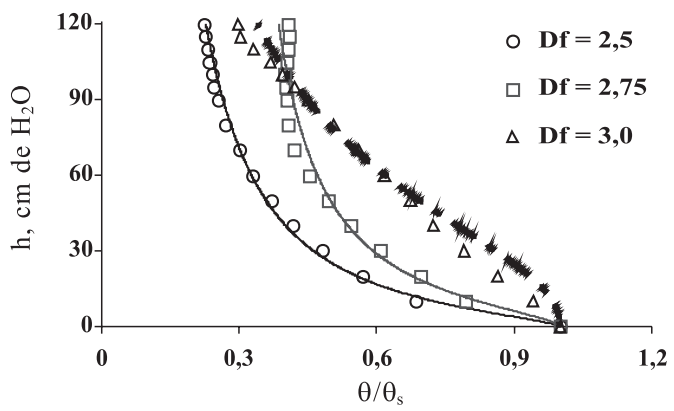

Figura 2. CRs para as amostras arenosas cujas granulometrias foram escolhidas com base na dimensão fractal de fracionamento $\left(D_{f}\right)$. Os pontos correspondem aos valores experimentais e as linhas representam as curvas ajustadas pelo modelo de van Genuchten.

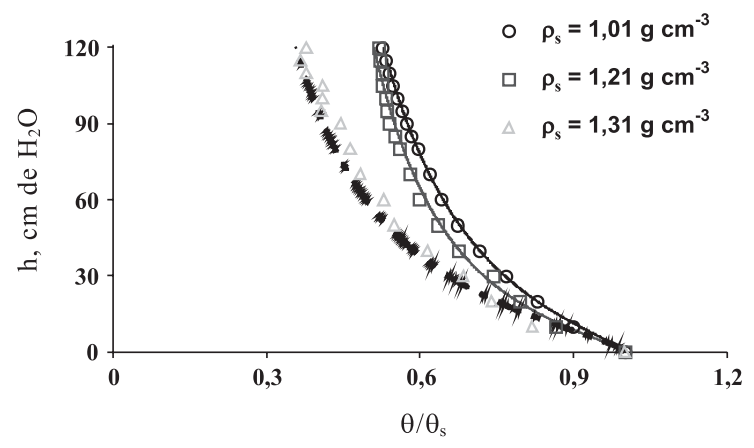

Figura 4. Curvas de retenção obtidas para os agregados de solo. Os pontos correspondem aos valores experimentais e as linhas representam as curvas ajustadas pelo modelo de van Genuchten.

Quadro 2. Informações sobre as características físicas e valores dos parâmetros obtidos no ajuste das curvas normalizadas para as amostras de solo arenoso investigadas

\begin{tabular}{cccccc}
\hline Amostra & $\boldsymbol{\rho}_{\mathbf{s}}{ }^{(1)}$ & $\boldsymbol{\varepsilon}^{(2)}$ & $\boldsymbol{\theta}_{\mathbf{r}}$ & $\boldsymbol{\theta}_{\mathbf{\theta}}$ & $\boldsymbol{\alpha}^{(3)}$ \\
\hline & $\mathrm{g} \mathrm{cm}^{-3}$ & $\%$ & $\mathrm{~cm}^{3} \mathrm{~cm}^{-3}$ & $\mathrm{~h}=0$ & $\mathrm{~cm}^{-1}$ \\
1 & $1,82 \pm 0,02$ & $34 \pm 2$ & 0,0020 & 0,9931 & 0,1298 \\
2 & $1,89 \pm 0,04$ & $29 \pm 2$ & 0,2323 & 0,9950 & 0,0965 \\
3 & $1,88 \pm 0,03$ & $29 \pm 2$ & 0,0010 & 0,9576 & 0,0197 \\
\end{tabular}

${ }^{(1)} \rho_{s}$ densidade do solo. ${ }^{(2)} \varepsilon$ porosidade do solo. ${ }^{(3)} \alpha$ e $n$ parâmetros teóricos de ajuste da equação de van Genuchten (1980).

Quadro 3. Informações sobre as características físicas e valores dos parâmetros obtidos no ajuste das $F C A_{n}$ para os agregados de LVd investigados

\begin{tabular}{|c|c|c|c|c|c|c|}
\hline \multicolumn{7}{|c|}{ Agregados de solo natural } \\
\hline d & $\rho_{\mathrm{s}}^{(1)}$ & $\varepsilon$ & $\theta_{\mathrm{r}}$ & $\theta / \theta_{\mathrm{s}}$ & $\alpha$ & $\mathbf{n}$ \\
\hline $\mathrm{cm}$ & $\mathrm{g} \mathrm{cm}^{-3}$ & $\%$ & $\mathrm{~cm}^{3} \mathrm{~cm}^{-3}$ & $\mathrm{~h}=0$ & $\mathrm{~cm}^{-1}$ & \\
\hline 1,621 & $1,31 \pm 0,01$ & $56 \pm 2$ & 0,0020 & 0,9799 & 0,0619 & 1,5080 \\
\hline 2,492 & $1,21 \pm 0,01$ & $59 \pm 2$ & 0,1248 & 0,9962 & 0,0768 & 1,3641 \\
\hline 5,477 & $1,01 \pm 0,01$ & $47 \pm 2$ & 0,0010 & 0,9967 & 0,0553 & 1,3270 \\
\hline
\end{tabular}

(1) $d$ representa um diâmetro médio dos agregados.

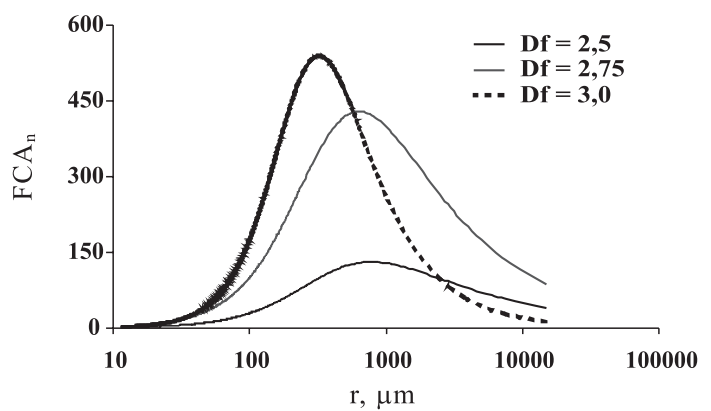

Figura 3. $F C A_{n}$ das amostras com granulometria dadas por $D_{f}$.

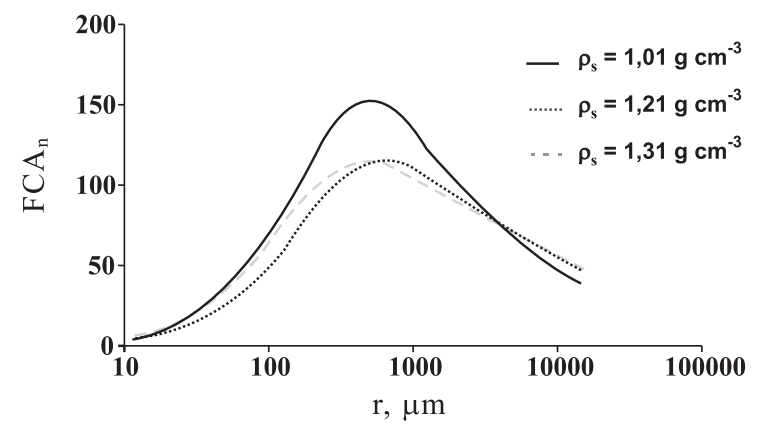

Figura 5. $F C A_{n}$ para os agregados de solo. 


\section{DISCUSSÃO}

Para realização dos experimentos, procurou-se reconstruir as amostras arenosas de modo que estas apresentassem densidades $\rho_{s}$ e porosidades $\varepsilon$ bastante próximas umas das outras (Quadro 2). Essa preocupação deveu-se ao fato de que, para a faixa de umidades investigadas neste experimento, a própria compactação das amostras poderia levar a alterações significativas nas CRs (Assouline et al., 1997; Ferrero \& Lipiec, 2000; Reichardt \& Timm, 2004). A densidade das amostras preparadas ficou em torno de $1,86 \mathrm{~g} \mathrm{~cm}^{-3}$, havendo uma variação máxima de $2,1 \%$ entre os valores de $\rho_{s}$ das amostras e o valor médio para este parâmetro.

O decréscimo no parâmetro $\alpha$ (Quadro 2) indica diminuição dos valores de sucção a serem aplicados para retirada de água das amostras. Esse fato pode ser observado pela análise das CRs das amostras (Figura 2). Observa-se que a aplicação de uma sucção de $3 \mathrm{kPa}$ (30 $\mathrm{cm}$ de altura de coluna de água) foi suficiente para reduzir a umidade da amostra de $D_{f}=2$ em cerca de $50 \%$. Já para a amostra de $D_{f}=3$ essa redução foi de apenas $10 \%$, para essa mesma sucção aplicada. Isso é explicado pelo fato de que o aumento de $D_{f}$ está ligado à presença de partículas de menores diâmetros nas amostras de solo (Comegna et al., 1998; Perfect, 1999). A presença dessas partículas menores implica o aparecimento de poros de menores diâmetros, os quais são esvaziados pela aplicação de maiores sucções na amostra (Tyler \& Wheatcraft, 1989).

Observou-se também aumento do parâmetro $n$ com o aumento de $D_{f}$. $\mathrm{O}$ aumento de $n$ indica que houve estreitamento da $F C A_{n}$. Portanto, além de se ter reduzido o diâmetro médio dos poros, a utilização de partículas de menores diâmetros na reconstrução das amostras reduziu a gama de poros que as constituíam (estreitamento da $F C A_{n}$ ). O estreitamento da $F C A_{n}$ indica também que o sistema poroso fica mais homogêneo com o aumento de $D_{f}$.

Os resultados obtidos para os agregados de solo mostram que suas CRs são pouco alterados pelos seus estados de compactação (Figura 4). Pode-se ver que há aumento de aproximadamente $13 \%$ no valor de $n$ com o aumento da densidade média dos agregados (Quadro 3). O valor de $\alpha$ oscila em torno de um valor médio de aproximadamente $0,0647 \mathrm{~cm}^{-1}$, mostrando que, para os agregados estudados, não houve diferenças sensíveis quanto a este parâmetro.

Com relação à $F C A_{n}$, observa-se que os agregados apresentam maior gama de poros (maior largura das $F C A_{n}$ ) (Figura 5) quando comparadas aos das amostras de solo arenoso (Figura 3). Isso demonstra que esse sistema poroso possui maior complexidade devido à presença de uma maior gama de poros de diferentes diâmetros em seu interior. É possível ainda observar que existe maior semelhança entre as distribuições de poros para os agregados estudados, o que se deve aos valores muito parecidos dos parâmetros de ajuste da equação de van Genuchten (Quadro 3).

O achatamento da distribuição de tamanho de poros para os agregados com maiores $\rho_{s}$ representa uma diminuição na freqüência dos poros com diâmetros entre 100 e $1.000 \mathrm{~mm}$, no caso dos agregados estudados. Esse resultado indica que o aumento de $\rho_{s}$ está relacionado a uma diminuição na quantidade de poros com diâmetros médios próximos ao valor mencionado anteriormente.

\section{CONCLUSÕES}

1. Pela adaptação do funil de Haines, foi possível obter curvas de retenção detalhadas das amostras investigadas de solos próximas à saturação.

2. A interpolação dos resultados utilizando-se a equação de van Genuchten forneceu as FCAs das amostras investigadas. Mais detalhadamente, da análise das FCAs obtidas foi possível verificar que: as amostras de agregados apresentaram maior complexidade em termos da gama de poros que as compõem; a variação da granulometria do solo arenoso está diretamente relacionada às modificações de suas propriedades de retenção de água - quanto menor o tamanho das partículas que constituem as amostras, menores são seus poros e, conseqüentemente, maior é a sucção necessária para se retirar a água delas; e a variação na densidade dos agregados investigados não alterou significativamente o comportamento das curvas de retenção na faixa de tensões estudada.

\section{LITERATURA CITADA}

ALEXANDER, L. \& SKAGGS, R.W. Predicting unsaturated hydraulic conductivity from the water characteristic. Trans. Am. Soc. Agric. Eng., 29:176-184, 1986.

AMÉZKETA, E. Soil aggregate stability: A review. J. Sust. Agric., 14:83-151, 1999.

ANDERSON, A.N.; MCBRATNEY, A.B. \& FITZPATRICK, E.A. Soil mass, surface, and spectral fractal dimensions photographs. Soil Sci. Soc. Am. J., 60:962-969, 1996.

ARYA, L.M. \& PARIS, J.F. A physicoempirical model to predict the soil moisture characteristic from particle-size distribution and bulk density data. Soil Sci. Soc. Am. J., 45:1023-1030, 1981.

ASSOULINE, S.; TAVARES-FILHO, J. \& TESSIER, D. Effect of compaction on soil physical and hydraulic properties: Experimental results and modeling. Soil Sci. Soc. Am. J., 61:390-398, 1997.

BROOKS, R.H. \& COREY, A.T. Hydraulic properties of porous media. Fort Collins, Colorado State University, 1964. (Hydrology Paper, 3) 
COMEGNA, V.; DAMIANI, P. \& SOMMELLA, A. Use of a fractal model for determining soil water retention curves. Geoderma, 85:307-323, 1998.

CRAWFORD, J.W.; MATSUI, N. \& YOUNG, I.M. The relation between the moisture-release curve and the structure of soil. Eur. J. Soil Sci., 46:369-375, 1995.

DEXTER, A.R.; KROESBERGEN, B. \& KUIPERS, H. Some mechanical properties of aggregates of top soils from the Ijsselmeer polders. 1. Undisturbed soil aggregates. Nether. J. Agric. Sci., 32:205-214, 1984.

EMPRESA BRASILEIRA DE PESQUISA AGROPECUÁRIA . EMBRAPA. Centro Nacional de Pesquisa de Solos. Manual de métodos de análise de solo. Rio de Janeiro, 1997. 212p.

FERRERO, A. \& LIPIEC, J. Determining the effect of trampling on soils in hillslope-woodlands. Inter. Agrophys., 14:9-16, 2000.

FUENTES, C.; VAUCLIN, M.; PARLANGE, J.Y. \& HAVERKAMP, R. A note on the soil-water conductivity of a fractal soil. Transp. Porous Media, 23:31-36, 1996.

FUENTES, C.; ANTONINO, A.C.D.; NETTO, A.M.; LIRA, C.A.B.O. \& CABRAL, J.J.S.P. Desenvolvimento de um modelo fractal para a estimativa da condutividade hidraúlica de solos não-saturados. R. Bras. Ci. Solos, 29:5160, 2005.

HATANO, R.; KAWAMURA, N.; IKEDA, J. \& SAKUMA, T. Evaluation of the effect of morphological features of flow path on solute transport by using fractal dimensions of methylene blue staining patterns. Geoderma, 53:31-44, 1992.

HAVERKAMP, R. \& PARLANGE, J.Y. Predicting the water retention curve from particle-size distribution I: Sandy soils without organic matter. Soil Sci. Soc. Am. J., 142:325$339,1986$.

HUANG, G.H. \& ZHANG, R.D. Evaluation of soil water retention curve with the pore-solid fractal model. Geoderma, 127:52-61, 2005.

JURY, A.W.; GARDNER, W.R. \& GARDNER, W.H. Soil physics. New York, John Wiley \& Sons, 1991. 328p.

KLUTE, A. Water retention: Laboratory methods. In: KLUTE, A., ed. Methods of soil analysis. I. Physical and mineralogical methods. Madison, Soil Science Society of America/American Society of Agronomy, 1986. p.635-662.

LEEDS-HARRISON, P.B. \& YOUNGS, E.G. Estimating the hydraulic conductivity of aggregates conditioned by different tillage treatments from sorption measurements. Soil Till. Res., 41:141-147, 1997.

LIBARDI, P.L. Dinâmica da água no solo. Piracicaba, Edição do Autor, 2000. 509p.

MANDELBROT, B. How long is the Coast Britain? Statistical self - Similarity and fractional dimension. Science, 156:636-638, 1967.
McKENZIE, B.M. \& DEXTER, A.R. Methods for studying the permeability of individual soil aggregates. J. Agric. Eng. Res., 65:23-28, 1996.

MUALEM, Y. Hydraulic conductivity of unsaturated soils: Prediction and formulas. In: KLUTE, A., ed. Methods of soil analysis. I. Physical and mineralogical methods. Madison, Soil Science Society of America/American Society of Agronomy, 1986. p.797-823.

PERFECT, E. Estimating soil mass fractal dimensions from water retention curves. Geoderma, 88:221-231, 1999.

PERFECT, E.; RASIAH, V. \& KAY, B.D. Fractal dimension of soil aggregate-size distributions calculated by number and mass. Soil Sci. Soc. Am. J., 56:1407-1409, 1992.

PERRIER, E.; BIRD, N. \& RIEU, M. Generalizing the fractal model of soil structure: The pore-solid fractal approach. Geoderma, 88:137-164, 1999

PIRES, L.F.; BACCHI, O.O.S. \& REICHARDT, K. Soil water retention curve determined by gamma-ray beam attenuation. Soil Till. Res., 82:89-97, 2005.

POULSEN, T.G.; MOLDRUP, P. \& JACOBSEN, O.H. Oneparameter models for unsaturated hydraulic conductivity. Soil Sci., 163:425-435, 1998

REICHARDT, K. \& TIMM, L.C. Solo, planta e atmosfera: Conceitos, processos e aplicações. São Paulo, Manole, 2004. $478 \mathrm{p}$.

SOIL AGGREGATES: Volume and density evaluation guide. Disponível em: < www.dot.state.co.us/DesignSupport/ field\%20Materials\%20Manual/CP\%2022.pdf.>. Acesso em 2005 .

SOIL SCIENCE SOCIETY OF AMERICA - SSSA. Glossary of soil science terms. Madison, 1997.

TYLER, S.W. \& WHEATCRAFT, S.W. Application of fractal mathematics to soil water retention estimation. Soil Sci. Soc. Am. J., 53:987-996, 1989.

TYLER, S.W. \& WHEATCRAFT, S.W. Fractal scaling of soil particle - Size distributions: Analysis and limitations. Soil Sci., 56:362-369, 1992.

van GENUCHTEN, M.Th. A Closed - Form equation for predicting the hydraulic conductivity of unsaturated soils. Soil Sci. Soc. Am. J., 44:892-898, 1980.

van GENUCHTEN, M.Th.; LEIJ, F.J. \& YATES, S.R. The RETC code for quantifying the hydraulic functions of unsaturated soils. Riverside, U. S. Salinity Laboratory, 1991. 85p.

van GENUCHTEN, M.Th. \& LEIJ, F.J. On estimating the hydraulic properties of unsaturated soils. Riverside, US Salinity Laboratory and Department of Soil and Environmental Sciences, University of California, 1992. $14 \mathrm{p}$.

ZHUANG, J.; NAKAYAMA, K.; YU, G.R. \& MIYAZAKI, T. Predicting unsaturated hydraulic conductivity of soil based on some basic soil properties. Soil Till. Res., 59:143-154, 2001. 